\title{
Safety of Drug-Eluting-Beads Transarterial Chemoembolization for Unresectable Hepatocellular Carcinoma in Patients with Transjugular Intrahepatic Portosystemic Shunt
}

\author{
Katherine Rief ${ }^{1} \quad$ Ali Pirasteh ${ }^{1} \quad$ Anuradha Shenoy-Bhangl ${ }^{2} \quad$ Suvranu Ganguli ${ }^{2,3} \quad$ Sanjeeva P. Kalva ${ }^{1}$ \\ ${ }^{1}$ Department of Radiology, University of Texas Southwestern \\ Medical Center, Dallas, Texas, United States \\ ${ }^{2}$ Department of Radiology, Beth Israel Deaconess Medical Center, \\ Boston, Massachusetts, United States

\begin{abstract}
Address for correspondence Ali Pirasteh, MD, Department of Radiology, University of Texas Southwestern Medical Center, 5323 Harry Hines Blvd., Dallas, TX 75390-9178, United States (e-mail: ali.pirasteh@utsouthwestern.edu).
\end{abstract}

${ }^{3}$ Department of Radiology, Massachusetts General Hospital, Boston, Massachusetts, United States

J Clin Interv Radiol ISVIR 2018;2:71-75

\begin{abstract}
Keywords

- transarterial chemoembolization

- hepatocellular carcinoma

- transjugular intrahepatic portosystemic shunt

Purpose To assess the safety of drug-eluting beads transarterial chemoembolization (DEB-TACE) for unresectable hepatocellular carcinoma (HCC) in patients with transjugular intrahepatic portosystemic shunt (TIPS).

Materials and Methods Records of patients with a patent TIPS who underwent DEBTACE for inoperable HCC from 2005 to 2014 at two institutions were reviewed. Patient demographics, number of procedures, extent of embolization (lobar vs. segmental), 30-day mortality, and postprocedure adverse events classified based on Common Terminology Criteria for Adverse Events (CTCAE V4.03) were recorded.

Results From the total of 10 patients included ( 6 male, average age: 59), 3 were classified as Child A and 7 as B. Tumor Barcelona clinic liver cancer (BCLC) stage was $A$ in three patients, $B$ in six, and $C$ in one. Patients underwent doxorubicin DEB-TACE with a median dose of $100 \mathrm{mg}$ (range: $50-100$ ). Number of TACE procedures per patient ranged from 1 to 4 (median: 1 ). Three (30\%) patients experienced toxicity grade 2 or higher immediately following the procedure. One patient had grade 3 toxicity of aspartate transaminase (AST), alanine transaminase (ALT), and bilirubin. Three patients had extended hospital stays beyond 48 hours due to adverse events, including edema, acute pancreatitis, and a hepatic abscess discovered a week after the procedure. All 10 patients were alive and discharged from the hospital at 30 days after the procedure. Conclusion DEB-TACE can be well tolerated by patients with a patent TIPS, given adequate liver function. Lobar DEB-TACE is associated with higher risk of complications.
\end{abstract}

\section{Introduction}

Drug-eluting beads transarterial chemoembolization (DEBTACE) allows tumor embolization and subsequent ischemic necrosis along with localized intra-tumoral drug delivery in patients with intermediate-stage hepatocellular carcinoma (HCC). DEB-TACE has been shown to be at least as effective as conventional chemoembolization..$^{1-3}$ In addition, it was found

received

September 14, 2017

accepted after revision

December 19, 2017

published online

June 20, 2018 to be safer than conventional TACE, especially in high-risk populations-patients with Child B cirrhosis and recurrent or bilobar HCC. ${ }^{4}$

Though cirrhosis serves as a well-known risk factor for HCC, the natural progression of disease leads to portal hypertension and its subsequent complications, such as any combination of variceal bleeds, recurrent ascites, and hepatic hydrothorax. Hence, many cirrhotic patients undergo

Copyright $@ 2018$ by Indian Society of Vascular and Interventional Radiology

License terms

10.1055/s-0038-1661001.

ISSN 2457-0214. 
a transjugular intrahepatic portosystemic shunt (TIPS) placement. After TIPS placement, there is significant shunting of the portal venous blood flow into the systemic veins with associated compensatory increase in the hepatic arterial blood flow to the normal liver parenchyma. Not uncommonly, these patients develop HCC after TIPS placement and represent a high-risk category for loco-regional therapies via the hepatic artery, as these therapies depend on preferential hepatic arterial supply to the tumor and preserved hepatopetal portal venous flow to normal liver. Some studies have demonstrated the safety of conventional TACE in patients with a preexisting TIPS. ${ }^{5,6}$ However, safety of DEB-TACE in this setting has not yet been studied. This study was designed to retrospectively assess the safety of DEB-TACE for inoperable HCC in patients with preexisting TIPS.

Table 1 Demographics of the study population

\begin{tabular}{|l|l|}
\hline Median age in years (range) & $58(39-74)$ \\
\hline Median MELD score (range) & $12(7-21)$ \\
\hline Child-Pugh-Turcotte class & Number of patients \\
\hline A & 3 \\
\hline B & 7 \\
\hline Background liver disease & Number of patients \\
\hline Viral hepatitis B & 2 \\
\hline Viral hepatitis C & 2 \\
\hline Hemochromatosis & 2 \\
\hline NASH & 1 \\
\hline Alcoholic cirrhosis & 5 \\
\hline $\begin{array}{l}\text { Duration of TIPS prior to } \\
\text { DEB-TACE }\end{array}$ & 4 days-17 years \\
\hline $\begin{array}{l}\text { Average tumor size } \\
\text { (range) }\end{array}$ & $2.8 \mathrm{~cm}$ \\
\hline
\end{tabular}

Abbreviations: DEB-TACE, drug-eluting beads transarterial chemoembolization; MELD, Model for End-Stage Liver Disease; NASH, nonalcoholic steatohepatitis; TIPS, transjugular intrahepatic portosystemic shunt.

\section{Materials and Methods}

\section{Patients}

Institutional review board approval was obtained, and all patient information was handled in compliance with HIPAA protocols. The authors retrospectively reviewed the medical records of all 10 patients with a functioning, patent TIPS who underwent DEB-TACE for unresectable HCC between 2005 and 2014 at two institutions. Patient demographics are summarized in -Table 1. Screening for HCC was done by ultrasound per routine clinical standard or by computed tomography (CT) or magnetic resonance imaging (MRI) for those with known lesions. All patients belonged to class A or $B$ using the Child-Pugh-Turcotte (CPT) classification system. Etiology of underlying liver disease was diverse, including alcoholic cirrhosis, nonalcoholic steatohepatitis (NASH), viral hepatitis $\mathrm{B}$, viral hepatitis $\mathrm{C}$, and hemochromatosis. Tumor and disease stage are described in - Table 2. The interval between placement of the TIPS and DEB-TACE procedure ranged from 4 days to 17 years.

\section{TIPS Procedure}

Four patients underwent TIPS placement for recurrent variceal bleed whereas the indication for one patient was recurrent ascites refractory to medical treatment. A VIATORR-covered stent graft (W.L. Gore \& Associates) was used in all five cases. Following the TIPS procedure, the portosystemic gradients were reported between 3 and $7 \mathrm{~mm} \mathrm{Hg}$. Five other patients underwent TIPS placement at outside institutions, and these medical records were unavailable for review.

\section{TACE Procedure}

Total 15 DEB-TACE procedures were performed in 10 patients (-Fig. 1), with a median of one procedure per patient (range: 1-4). Micrometer-sized (100-300 $\mu \mathrm{m}$ ), doxorubicin-loaded polyvinyl alcohol hydrogel particles (LC Beads, BTG International Ltd.) were used as the embolization material, with an average doxorubicin dose of $75 \mathrm{mg}$ per procedure (range: 50-150 mg, median: $100 \mathrm{mg}$ ). Nine selective

Table 2 Tumor characteristics and staging at time of initial TACE

\begin{tabular}{|c|c|c|c|c|c|c|}
\hline Patient & $\begin{array}{l}\text { Number of } \\
\text { lesions }\end{array}$ & Liver segment/lobe & $\begin{array}{l}\text { Largest tumor } \\
\text { diameter }(\mathrm{cm})\end{array}$ & $\begin{array}{l}\text { MELD } \\
\text { score }\end{array}$ & $\begin{array}{l}\text { CPT } \\
\text { score: class }\end{array}$ & $\begin{array}{l}\text { BCLC } \\
\text { stage }\end{array}$ \\
\hline 1 & 1 & IVB & 6.5 & 14 & $8: B$ & $\mathrm{~B}$ \\
\hline 2 & 1 & V/VIII & 1.9 & 13 & 8: $B$ & B \\
\hline 3 & 1 & IVB & 3.3 & 11 & 8: B & B \\
\hline 4 & 1 & VII/VIII & 1.8 & 14 & 8: B & B \\
\hline 5 & 2 & III, VIII & $1.7,2.0$ & 11 & $6: A$ & $\mathrm{~B}$ \\
\hline 6 & Multiple & Right lobe & 2.8 & 12 & $6: A$ & B \\
\hline 7 & Multiple & IVB & 2.3 & 7 & 5: A & $A$ \\
\hline 8 & 1 & VII/VIII & 2.0 & 12 & 8: B & A \\
\hline 9 & 1 & II & 2.8 & 21 & 8: B & A \\
\hline 10 & 1 & VIII & 2.5 & 13 & 8: $B$ & C \\
\hline
\end{tabular}

Abbreviations: BCLC, Barcelona clinic liver cancer; CPT, Child-Pugh-Turcotte; MELD, Model for End-Stage Liver Disease; TACE, transarterial chemoembolization. 


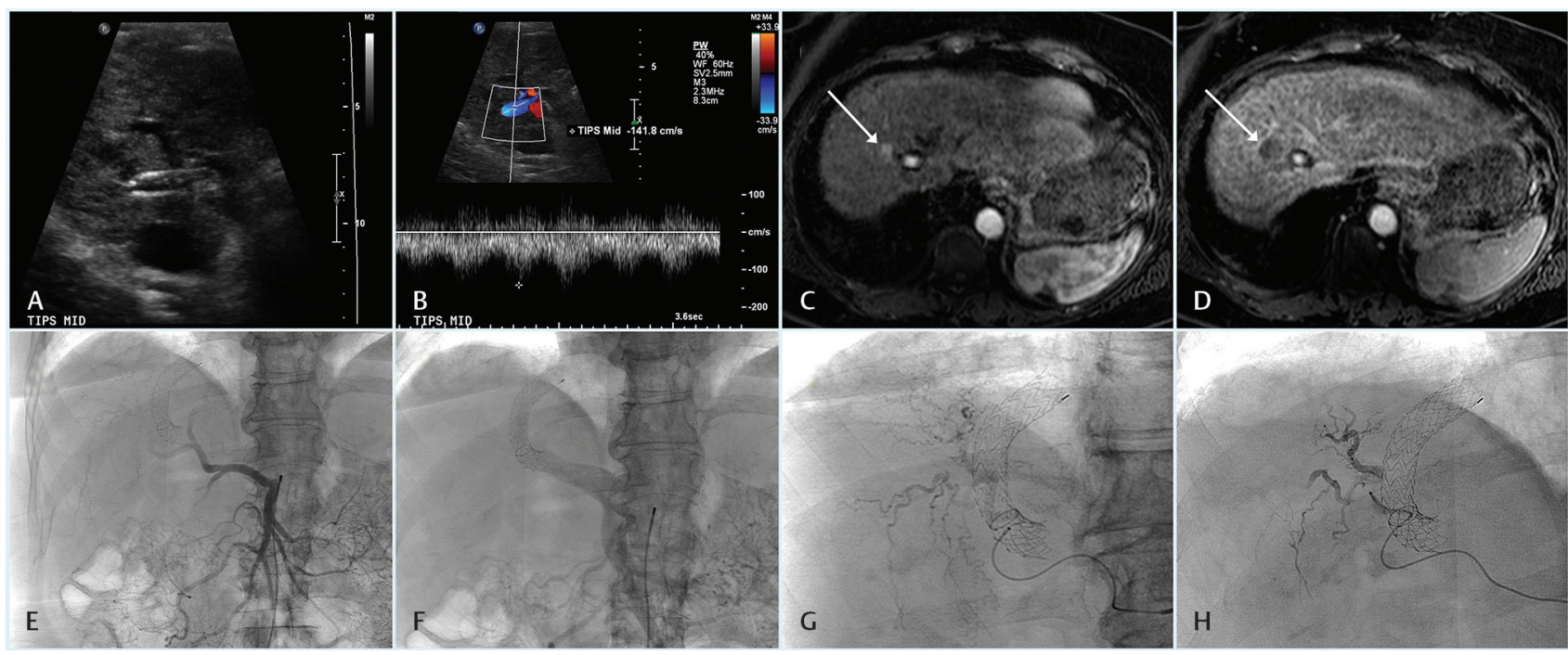

Fig. 1 A 71-year-old woman with NASH cirrhosis who underwent TIPS placement for recurrent variceal bleed. (A, B) Routine screening liver ultrasound demonstrates a patent TIPS with normal waveform. (C, D) A lesion suspected on the screening sonogram was then further evaluated by an MRI of the abdomen with/without IV contrast, confirming a $1.8-\mathrm{cm}$ lesion with hyperenhancement on the arterial phase (C, arrow) and washout on the delayed venous phase (D, arrow), consistent with HCC. Patient was referred to interventional radiology for TACE. (E-H) A superior mesenteric angiogram demonstrated a replaced common hepatic artery $(\mathbf{E})$ with delayed follow-through depicting a patent main portal vein and a patent TIPS (F). Selective angiogram of a segment VIII hepatic artery with a blush corresponding to the lesion on MRI (G), which was embolized with $2 \mathrm{~mL}$ of drug-eluding beads (100-300 $\mu \mathrm{m}$ ) loaded with $75 \mathrm{mg}$ of doxorubicin. Postembolization angiogram (H) demonstrated continued antegrade flow without a tumor blush.

embolization procedures were performed, guiding the microcatheter into a second- or third-order artery nearest to the tumor. The remaining six DEB-TACE procedures were conducted in a lobar fashion. The number of DEB-TACE per patient ranged from 1 to 4 , with a median of 1 DEB-TACE per patient.

\section{Study Outcomes}

Assessment of hepatotoxicity as well as other complications following DEB-TACE was based on the occurrence of hepatobiliary and other adverse events defined using the National Cancer Institute Common Terminology Criteria for Adverse Events (NCI CTCAE) version 4.03. ${ }^{7}$ Overall survival was documented from time of last DEB-TACE to endpoints of death, transplant, or January 1, 2015.

\section{Results}

There were no deaths within 30 days of the procedure. Median postprocedure hospital stay following the DEB-TACE was 2 days, (range: $1-8$ ). One patient who underwent lobar embolization required a prolonged 5-day hospitalization for acute, necrotizing pancreatitis and upper gastrointestinal (GI) bleed (CTCAE grade 4 toxicity). Another patient who underwent lobar embolization required an 8-day hospitalization for CTCAE grade 4 liver toxicity. One patient who underwent segmental embolization experienced a normal postprocedure stay, however was admitted 2 weeks later for sepsis and was found to have a hepatic abscess. One patient experienced transient azotemia with serum creatinine elevated up to twice the normal limit with no dialysis requirement, which may have been related to contrast-induced nephrotoxicity.
Less severe adverse events that did not result in prolonged hospital stay (CTCAE grades 1 and 2) are listed in - Table 3.

Mean follow-up period following DEB-TACE was 10 months (2-30 months). Patients were followed through January of 2015. Five of the 10 patients were still alive at last follow-up. The patients who were deceased had a median survival of 6 months following the last TACE procedure. Cause of death for all patients was progression of disease with liver and/or multiorgan failure. Median survival following the last TACE for those patients still alive at last follow-up was 14.6 months. In one case transplant was considered the endpoint for overall survival.

\section{Discussion}

A retrospective study by Kohi et $\mathrm{al}^{8}$ reviewed 158 patients who underwent conventional TACE for treatment of HCC; 10 patients had a preexisting TIPS. The study concluded that the patient population with a patent TIPS were more likely to develop significant hepatotoxicity after TACE than a comparable group of patients without a TIPS. They noted a 2 -fold increase in the rate of severe hepatobiliary dysfunction and a 1.5-fold increase in requirement of liver transplant 1 year after TACE. Although multiple prior isolated case reports or small series have demonstrated successful results, ${ }^{5,6,9}$ detailed evaluation of these studies reveals nonuniformity across these studies in terms of the embolization agent used, the technique, including dose of embolization material used, selected patient population, and the definition of successful outcome.

Based on the reported findings, the authors conclude that DEB-TACE is a well-tolerated option for unresectable 
Table 3 Patient information, procedure details, and adverse events

\begin{tabular}{|l|l|l|l|l|l|l|l|}
\hline Patient & $\begin{array}{l}\text { Age } / \\
\text { sex }\end{array}$ & $\begin{array}{l}\text { Indication } \\
\text { for TIPS }\end{array}$ & $\begin{array}{l}\text { No. of TACE } \\
\text { procedures }\end{array}$ & $\begin{array}{l}\text { Lobar vs. } \\
\text { segmental } \\
\text { DEB-TACE }\end{array}$ & $\begin{array}{l}\text { Serum AFP } \\
\text { level prior to } \\
\text { first DEB-TACE }\end{array}$ & $\begin{array}{l}\text { Hospital stay } \\
\text { post each DEB- } \\
\text { TACE (days) }\end{array}$ & $\begin{array}{l}\text { Significant adverse } \\
\text { events (CTCAE } \\
\text { grade 2 2) }\end{array}$ \\
\hline 1 & $39 / \mathrm{M}$ & Varices & 1 & Segmental & 2 & 1 & $\begin{array}{l}\text { Transient elevation of } \\
\text { alkaline phosphatase (1) }\end{array}$ \\
\hline 2 & $56 / \mathrm{M}$ & Varices & 1 & Segmental & 3 & 2 & None \\
\hline 3 & $52 / \mathrm{F}$ & Ascites & 1 & Lobar $^{\mathrm{a}}$ & 8 & 5 & $\begin{array}{l}\text { Necrotizing pancreatitis (4) } \\
\text { Liver toxicity (4) } \\
\text { Upper Gl bleed (3) }\end{array}$ \\
\hline 4 & $71 / \mathrm{F}$ & Varices & 1 & Segmental & 19.3 & 1 & $\begin{array}{l}\text { None immediate } \\
\text { Hepatic abscess 2 wk } \\
\text { postprocedure (3) }\end{array}$ \\
\hline 5 & $56 / \mathrm{M}$ & Varices & 2 & Segmental & 4.2 & 1,1 & $\begin{array}{l}\text { Transient bilirubin rise (2) } \\
\text { Transient mild } \\
\text { transaminitis (1) }\end{array}$ \\
\hline 6 & $74 / \mathrm{F}$ & Varices & 4 & Lobar & 3.3 & $2,3,2,2$ & None \\
\hline 7 & $50 / \mathrm{F}$ & Varices & 1 & Lobar & 4.4 & 8 & Liver toxicity (4) \\
\hline 8 & $60 / \mathrm{M}$ & Varices & 2 & Segmental & 150.9 & 2,2 & None \\
\hline 9 & $70 / \mathrm{M}$ & Varices & 1 & Segmental & 27.0 & 2 & Transient azotemia (2) \\
\hline 10 & $64 / \mathrm{M}$ & Varices & 1 & Segmental & 136.5 & 2 & None \\
\hline
\end{tabular}

Abbreviations: AFP, $\alpha$-fetoprotein ( $\mu \mathrm{g} / \mathrm{L}$ ); CTCAE, Common Terminology Criteria for Adverse Events; DEB-TACE, drug-eluting beads transarterial chemoembolization; GI, gastrointestinal; TIPS, transjugular intrahepatic portosystemic shunt.

'Lobar TACE in patient 3 was performed due to the challenging nature of the anatomy, where the segmental artery could not be selected.

bLobar TACE in patient 7 was performed as the preembolization angiogram demonstrated arterial blushes in segments II and III, thus the left lobe was treated.

HCC in patients with preexisting TIPS. Complications leading to prolonged in-hospital course are more often seen in patients who receive lobar embolization, and supportive measures were adequate for inpatient management. Three of 10 patients had significant adverse events requiring prolonged hospitalization or readmission, two of which underwent lobar embolization. This is likely secondary to the less spatially specific nature of lobar embolization, inducing a higher ischemic burden than the more targeted segmental technique. Given the presence of the TIPS and reduced portal venous blood flow, the liver is largely dependent on arterial flow to perfuse the tissue. Further compromising this flow by embolizing an entire lobe of the liver during TACE may induce ischemia within a vulnerable liver, resulting in hepatotoxicity. Thus, caution should be taken prior to performing lobar DEB-TACE in patients with TIPS. In addition, other options such as yitrium-90 radioembolization can be considered as a potential alternative.

Limitations of the study include its retrospective nature and a small cohort. Owing to the small cohort, no statistical analysis was performed, as it would lack sufficient power to achieve statistically meaningful conclusions. Another limitation of the study is unavailability of follow-up patient information to evaluate for efficacy of treatment in addition to its safety.

\section{Conflict of Interest}

Dr. Kalva receives royalties from Springer and Elsevier, is an investor in Althea healthcare, personal fees from Koo
Foundation, Taiwan, personal fees from General Electric, and is an International Editor for the Journal of Clinical Interventional Radiology ISVIR (Indian Society of Vascular and Interventional Radiology), outside the submitted work.

\section{References}

1 Nicolini D, Svegliati-Baroni G, Candelari R, et al. Doxorubicin-eluting bead vs conventional transcatheter arterial chemoembolization for hepatocellular carcinoma before liver transplantation. World JGastroenterol2013;19(34):5622-5632

2 Huang K, Zhou Q, Wang R, Cheng D, Ma Y. Doxorubicin-eluting beads versus conventional transarterial chemoembolization for the treatment of hepatocellular carcinoma. J Gastroenterol Hepatol 2014;29(5):920-925

3 Zhou X, Tang Z, Wang J, et al. Doxorubicin-eluting beads versus conventional transarterial chemoembolization for the treatment of hepatocellular carcinoma: a meta-analysis. Int J Clin Exp Med 2014;7(11):3892-3903

4 Prajapati HJ, Dhanasekaran R, El-Rayes BF, et al. Safety and efficacy of doxorubicin drug-eluting bead transarterial chemoembolization in patients with advanced hepatocellular carcinoma. J Vasc Interv Radiol 2013;24(3):307-315

5 Hsin IF, Hsu CY, Huang HC, et al. Liver failure after transarterial chemoembolization for patients with hepatocellular carcinoma and ascites: incidence, risk factors, and prognostic prediction. J Clin Gastroenterol 2011;45(6):556-562

6 Kang JW, Kim JH, Ko GY, Gwon DI, Yoon HK, Sung KB. Transarterial chemoembolization for hepatocellular carcinoma after transjugular intrahepatic portosystemic shunt. Acta Radiol 2012;53(5):545-550 
7 Institute NC. Common Terminology Criteria for Adverse Events. June 14, 2010. http://ctep.cancer.gov/protocolDevelopment/electronic_applications/ctc.htm

8 Kohi MP, Fidelman N, Naeger DM, LaBerge JM, Gordon RL, Kerlan RK Jr. Hepatotoxicity after transarterial chemoembolization and transjugular intrahepatic portosystemic shunt: do two rights make a wrong? J Vasc Interv Radiol 2013;24(1):68-73
9 Sakaguchi H, Uchida H, Maeda M, et al. Combined transjugular intrahepatic portosystemic shunt and segmental Lipiodol hepatic artery embolization for the treatment of esophagogastric varices and hepatocellular carcinoma in patients with cirrhosis: preliminary report. Cardiovasc Intervent Radiol 1995;18(1):9-15 